\title{
Attention-based texture segregation
}

\author{
THOMAS V. PAPATHOMAS \\ Rutgers University, Piscataway, New Jersey \\ ANDREI GOREA \\ Laboratoire de Psychologie Expérimentale, CNRS, Paris, France \\ and René Descartes University, Paris, France \\ and \\ AKOS FEHER and TIFFANY E. CONWAY \\ Rutgers University, Piscataway, New Jersey
}

\begin{abstract}
Luminance- or color-defined $\pm 45^{\circ}$-oriented bars were arranged to yield single-feature or doubleconjunction texture pairs. In the former, the global edge between two regions is formed by differences in one attribute (orientation, or color, or luminance). In the color/orientation double-conjunction pair, one region has $+45^{\circ}$ red and $-45^{\prime \prime}$ green textels, the other $-45^{\circ}$ red and $+45^{\circ}$ green textels (the luminance/orientation double-conjunction pair is similar); such a pair contains a single-feature orientation edge in the subset of red (or green) textels, and a color edge in the subset of $+45^{\circ}$ (or $-45^{\circ}$ ) textels. We studied whether edge detection improved when observers were instructed to attend to such subsets. Two groups of observers participated: in the test group, the stimulus construction was explained to observers, and they were cued to attend to one subset. The control group ran through the same total number of sessions without explanations/cues. The effect of cuing was weak but statistically significant. Feature cuing was more effective for color/orientation than for luminance/orientation conjunctions. Within each stimulus category, performance was nearly the same no matter which subset was attended to. On average, a global performance improvement occurred over time even without cuing, but some observers did not improve with either cuing or practice. We discuss these results in the context of oneversus two-stage segregation theories, as well as by reference to signal enhancement versus noise suppression. We conclude that texture segregation can be improved by attentional strategies aimed to isolate specific stimulus features.
\end{abstract}

The improvement of our visual performance through priming one of the attributes (color, luminance, size, shape, location, etc.) that characterize a target stimulus embedded in a multidimensional "distracting" field has been documented for decades (e.g., Green \& Andersen, 1956; Smith, 1962). Most of the literature has assessed this priming effect in terms of a reduction in the reaction time for the cued trials, but sensitivity enhancement has also been claimed (e.g., Bravo \& Nakayama, 1992; Humphreys, 1981; Maljkovic \& Nakayama, 1994; Raymond, O’Donnell, \& Tipper, 1997; Tsal \& Lavie, 1988). Gen-

This work was supported by Grant DRET $94-047$ to A.G., and J. S. McDonnell Foundation Grant 95-60 to T.V.P. and A.F. Patrick Cavanagh was very helpful in discussing attention-related issues with one of us. We thank Janos Geier and Jim McGowan for helping with the statistics, and Ilona Kovács for helpful suggestions. John Duncan and Jeremy Wolfe provided invaluable feedback as reviewers to improve the contents and style of this paper. Part of this study was presented at the 1996 Meeting of the Association for Vision Research and Ophthalmology, Fort Lauderdale, Florida. Correspondence should be addressed to T.V. Papathomas, Rutgers University, Laboratory of Vision Research, Psychology Building-Busch Campus, Piscataway, NJ 08854 (e-mail: papathom@zeus.rutgers.edu)

-Accepted by previous editor, Myron L. Braunstein erally, attribute-priming experimental designs involve an odd-man-out task. It is less clear whether or not attribute priming is equally efficient in texture discrimination tasks.

One way or another, the role of attribute priming is to reduce the size of the relevant attribute space within which the target is to be found. Phrased differently, attribute priming decreases the uncertainty that characterizes search in a multiattribute space. Manipulating uncertainty has been the preferred method in assessing attentional effects in early-vision experiments (see Pelli, 1985, for a review). The general claim of this literature is that any uncertainty-related sensitivity effect is evidence that the observer can somehow selectively monitor (or attend to) the sensory space circumscribed by the manipulated uncertainty. In a circular manner, the effectiveness of an uncertainty manipulation (explicitly, through priming, or implicitly, through repetition; e.g., Bravo \& Nakayama, 1992) was taken as evidence for the existence of distinct sensory mechanisms precisely because they could be monitored independently (e.g., Davis, Kramer, \& Graham, 1983; Kramer, Graham, \& Yager, 1985). The main unanswered question in this literature is whether the observed effects are to be accounted for at the coding level (signal enhancement) or at the decision level (e.g., Palmer, 1994). Manipulation of uncertainty (through priming or other- 
wise) may be associated with a change in the number of noise sources to be considered at the decision level, thereby affecting a sensitivity index such as $d^{\prime}$. Recent studies favor this noise suppression interpretation (Dosher \& Lu, 1997; Shiu \& Pashler, 1994a, 1994b), but evidence supporting the signal enhancement hypothesis also exists (Raymond et al., 1997).

Assessing the extent to which an observer can attend to an arbitrary perceptual attribute/dimension may be achieved without manipulating uncertainty. It is possible to design stimuli defined within a multiattribute space in such a way that each of their defining features carries sufficient information for the task to be feasible, while their actual mixture renders the task difficult or even impossible. One can then inquire into the observer's capacity to extract one such relevant feature in the presence of the remaining ones so as to improve his/her performance.

A case in point is the double-conjunction stimulus (Treisman \& Gelade, 1980; Wolfe, Cave, \& Franzel, 1989) generalized to study texture segregation (Gorea \& Papathomas, 1991, 1993; Wolfe, 1992). Figure 1a illustrates a typical texture pair whose edge is defined by the double conjunction of luminance polarity (dark/bright) and orientation $\left( \pm 45^{\circ}\right)$ : the left-hand texture is composed of bright $-45^{\circ}$-oriented, and dark $+45^{\circ}$-oriented textels, while the right-hand texture contains bright $+45^{\circ}$ - and dark $-45^{\circ}$-oriented textels. The reader would probably agree that finding the texture edge in this stimulus is a quite difficult task. ' However, if he/she manages, one way or another, to "isolate" the bright textels, the segregation task should be trivial, and the orientation-defined edge will "pop out," as in Figure 1c. In a dual way, the observer may "focus" on, say, the $-45^{\circ}$ elements and find the polaritydefined edge, as in Figure 1d. This two-stage "attentional" strategy has been proposed as a general algorithm for resolving double-conjunction-defined targets (FriedmanHill \& Wolfe, 1995; Treisman \& Gelade, 1980; Treisman \& Sato, 1990; Wolfe, 1994; Wolfe et al., 1989).

In the case of visual search, it is easy to imagine that detection of a double-conjunction-defined target can be guided by knowledge of one of its defining features, particularly if eye movements are allowed (Egeth, Virzi, \& Garbart, 1984; Friedman-Hill \& Wolfe, 1995). It is less obvious, however, if and how one might select a given feature map in a double-conjunction texture pair so as to transform its edge into a single-feature-defined one. It has been indeed shown that visual search and texture segmentation are not equivalent tasks (Wolfe, 1992). The literature provides no direct evidence in favor of the proposed two-stage strategy.

The advantage of an attentive map selection is less obvious than it seems. If one is to assume the existence of four visual "maps"- $\mathrm{ON}_{+45^{\circ}}, \mathrm{ON}_{45^{\circ}}, \mathrm{OFF}_{+45^{\circ}}, \mathrm{OFF}_{-45^{\circ}}$ (e.g., Duncan \& Humphreys, 1992) 2 $^{2}$-the extraction of the texture edge by selective attention to specific maps for the case illustrated in Figure la would consist of isolating any pair sharing one attribute- say, the "ON" map-and then extracting the edge defined by the second attribute within this map - that is, orientation. By doing so, however, edge information carried by the orientation contrast within the "OFF" map is suppressed. The selection process should then seem to decrease rather than increase performance. One possible way to account for a performance improvement by such a selection technique is to assume the existence of at least four additional maps: nonoriented $\mathrm{ON}$ and $\mathrm{OFF}$ units and polarity nonspecific $+45^{\circ}$ and $-45^{\circ}$ units. ${ }^{3}$ Because their activation is irrelevant to the segmentation task in the double-conjunction texture pair of Figure 1, it plays the role of noise. As a consequence, while "attending" to two of the four relevant maps (the ones that are tuned to both polarity and orientation) will entail a reduction of the signal strength, it will also discard the four "noise maps" (the ones that are tuned only to polarity or only to orientation). The increase in overall sensitivity could mean that the suppression of some signal sources is more than compensated for by the concomitant suppression of the noise sources.

It should be noted that, although described here as a two-stage process, the selection scheme just described is equivalent to a one-stage process whereby $\mathrm{ON}_{+45^{\circ}}$ (or $\mathrm{ON}_{-45^{\circ}}$ ) maps are selected from the very start. Such units will signal the double-conjunction edge on their own. In any event, the putative increase in the discriminability of such an edge that results from "focusing" on one of its constituent features remains to be confirmed experimentally. In the affirmative, the next question to be answered is whether or not some attributes can be monitored more readily than others can.

The dimensions under focus in the present study are color, luminance, and orientation. Observers were asked to discriminate double-conjunction texture edges prior to and after the building principle of the stimuli was explained to them, and they were instructed to "pay attention" to only one value of their constituent elements - red, green, dark, bright, $\pm 45^{\circ}$-and extract the edge within that subpopulation of textels. In order to present meaningful comparisons between potential performance gains yielded by cuing any of these features, intra-attribute contrasts must be set at an equivalent, or at least at a known, discriminability level. The assessment of equivalent contrasts across stimulus dimensions is of main concern in studies that compare their efficiencies in any psychophysical task (such as direction discrimination, Agonie \& Gorea, 1993; Gorea \& Papathomas, 1989; texture segregation, Gorea \& Papathomas, 1991; Kingdom, Moulden, \& Collyer, 1992; stereo, Papathomas, Kovács, Gorea, \& Julesz, 1995; or depth perception in general, Landy, Maloney, Johnston, \& Young, 1995).

\section{METHOD}

\section{General}

Before describing in detail the stimul1 and procedures, we present the rationale and purposes of the various experments that are reported in this paper. The main experiments dealt with texture pairs that were segregated by the double conjunction of etther luminance polarity and orientation (Figure la) or color and orienta- 
a

$$
\text { DCj } 100 \%
$$

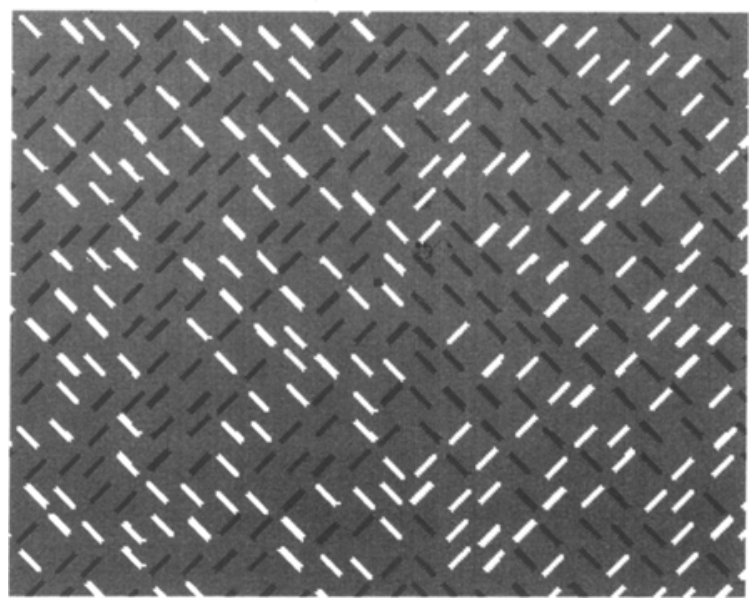

c

\section{All bright}

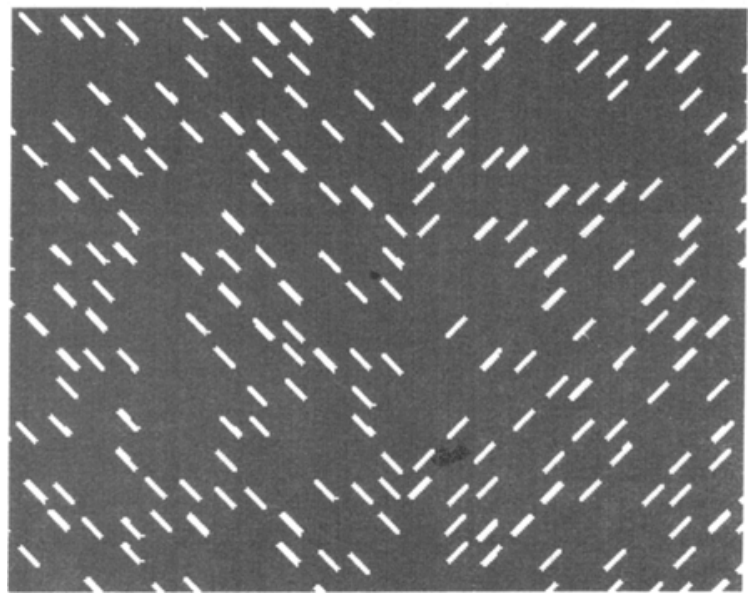

b DCj $50 \%$

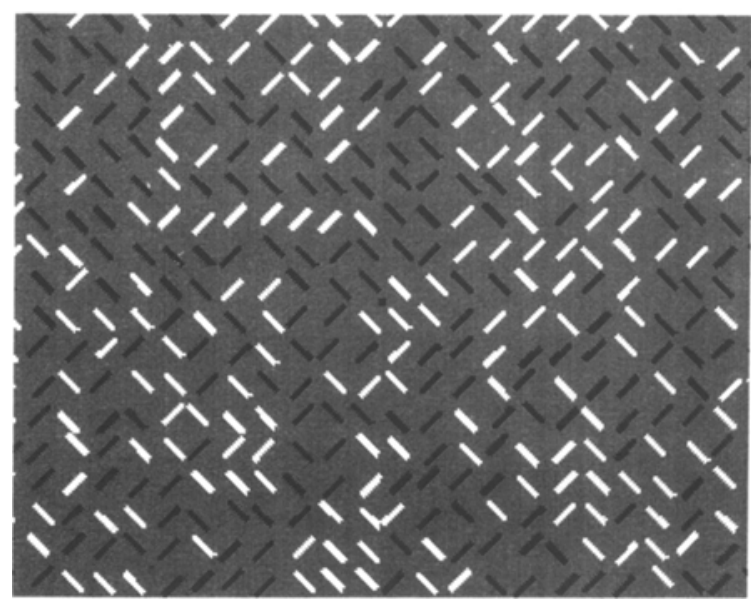

d
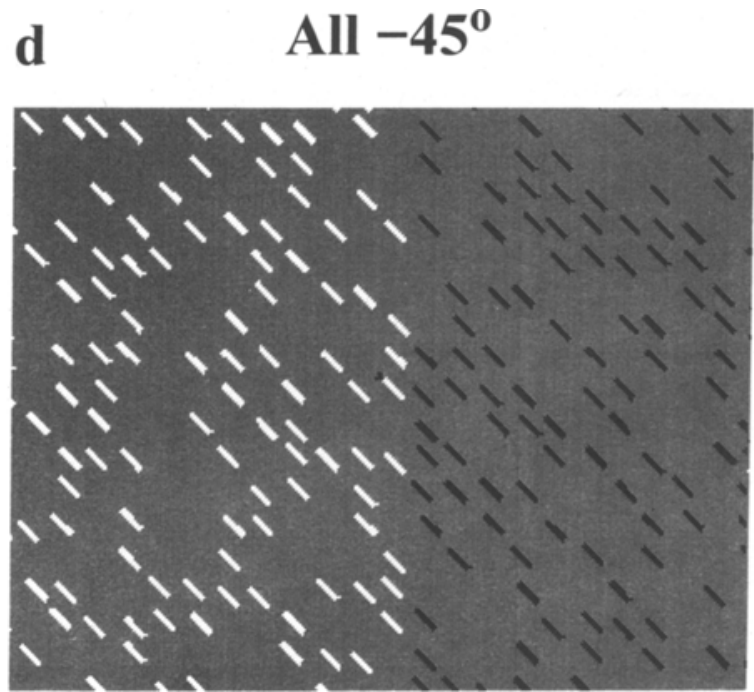

Figure 1. Luminance/orientation double-conjunction texture pair as used in this study. For the color/orientation conjunction, red and green textels replace dark and bright textels, respectively. The top panels show a $100 \%$ (a) and a $50 \%$ (b) signal double-conjunction texture pair (see text) with a vertical edge. The bottom panels were derived from panel a by selecting a subset of textels that observers could "isolate" to detect the global edge: (c) the subgroup of bright textels; (d) the subgroup of the $-45^{\circ}$ textels.

tion. Two groups participated in the main experiments: Observers in the test group were given cues on what feature to attend to, in order to extract the texture edge, after an initial precue phase; observers in the control group were never given such cues. This setup was designed to isolate any difference in performance that might have been due to attentional strategies. Three secondary experiments were conducted to fine-tune the stimuli for various purposes: The first was designed to obtain equivalent contrasts for the luminance polarity and for the color stimuli. The second was intended to establish equiluminant conditions in the color stimuli. The thırd was to obtain a "unique-yellow" background that would equate the chromatic contrast of the red and green textels.

\section{Stimuli}

Stimuli were displayed on a Sony GDM-17E11 RGB monitor, 1,280 pixels wide and 1,024 pixels high under the control of an Indy Silicon Graphics workstation. The screen subtended $21.8^{\circ} \times 17.1^{\circ}$ at a distance of $76 \mathrm{~cm}$ in all experiments. All experiments were run in a dimly illuminated room.

Main and equivalent-contrast experiments. The stimuli consisted of $+45^{\circ}$ and $/$ or $-45^{\circ}$ textels, $5.4^{\prime}$ ( 5 pixels) wide and $35.4^{\prime}$ (33 pixels) long, presented on a yellow background (chromatic CIE coordinates averaged across observers: $0.413,0.451$ ) of $27 \mathrm{~cd} / \mathrm{m}^{2}$. Luminance-defined textels were isochromatic to the background and yielded either variable or fixed (at $\pm 40 \%$ ) luminance contrast in the equivalent-contrast or main experiments, respectively. Chromaticdefined textels were equiluminant to the background and differed in chromaticity (red: $0.554,0.357$; green: $0.277,0.542$ ). The average center-to-center intertextel distance was $46.2^{\prime}$. In the main experiment, textels were arranged so as to yield vertical or horizontal edges defined by the conjunction of color and orientation or of luminance and orientation (Figure 1). In the equivalent-contrast experiments, they were arranged so as to yield texture pairs that were segregated by differences in one attribute, while the two values of 
a

\section{LxO 100\%}

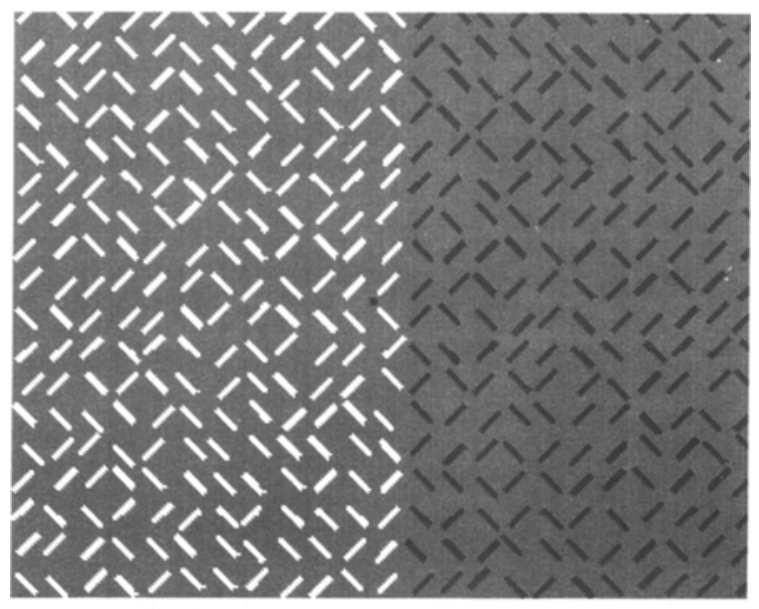

c

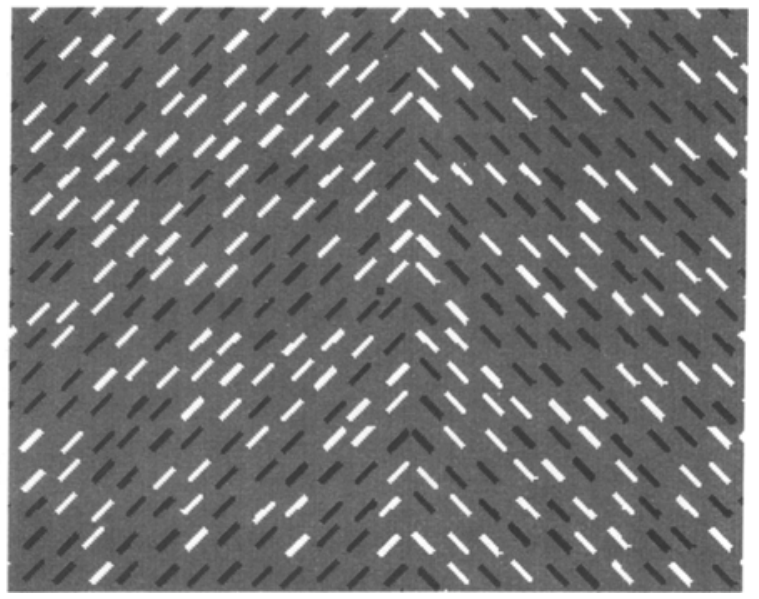

b $\mathrm{LxO} 50 \%$

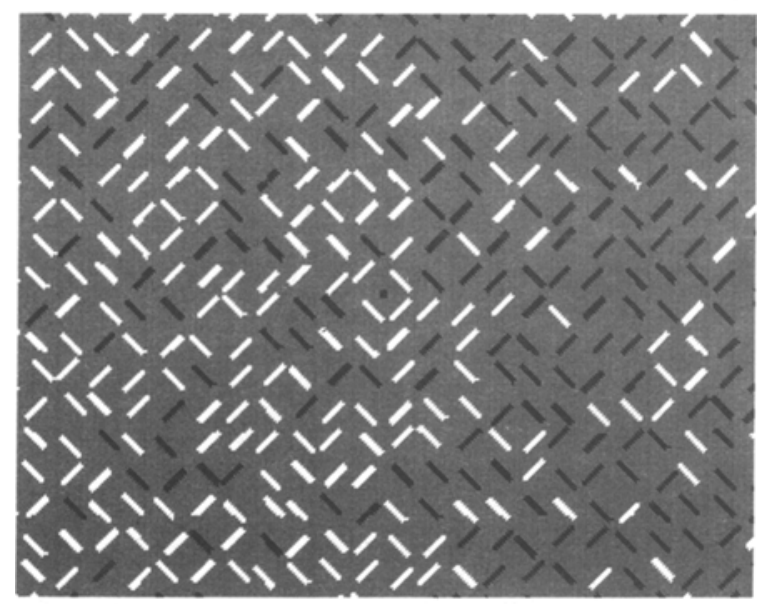

d

OxL 50\%

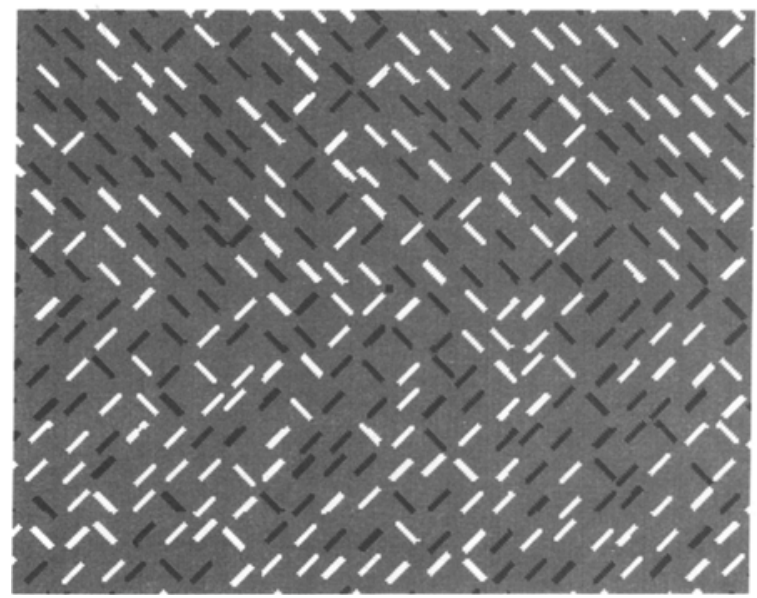

Figure 2. Luminance polarity $(a, b)$ and orientation $(c, d)$ single-feature texture pairs with vertical edges. Single-feature color configurations are obtained if the dark and bright textels are replaced with equiluminant red and green ones, respectively. Left- and right-hand panels show configurations involving $100 \%(a, c)$ and $50 \%(b, d)$ signal.

the other attribute were randomized throughout the image. The segregatıng features were as follows: (1) luminance, as shown in Figure $2 \mathrm{a} ;(2)$ color, similar to Figure $2 \mathrm{a}$, with dark and bright substituted by red and green, respectively; (3) orientation, with polarity randomized, as shown in Figure 2c; and (4) orientation, with color randomized, analogous to Figure $2 \mathrm{c}$ (see Gorea \& Papathomas, $1989,1991,1993)$. In the terminology used in the search literature, these stimuli are of the single-feature type. The horizontal or vertical edge of both types of texture pairs was randomly jagged within a spatial range of 2 textels, and its mean position was also randomized about the center of the screen within a range of 2 textels. Such a randomization ( 4 textels, or $1.54^{\circ}$ overall) was meant to minimize the chances that observers might infer the orientation of the edge by means of systematic eye movements. To this same effect, observers were repeatedly reminded to maintain fixation on a central, conspicuous cross. Single-feature and double-conjunction stimul 1 were presented for only 417 and $694 \mathrm{msec}$, respectively (shorter durations yielded performances close to chance level).

Equiluminance stimuli. The stımuli were $16.1^{\prime} \times 2.8^{\circ}$ periodically displayed red, green, and/or yellow bars on a yellow background. They were animated at an $18-\mathrm{Hz}$ rate. A detailed account of their spatiotemporal configuration can be found in Gorea, Papathomas, \& Kovács (1993).

Unique yellow stimuli consisted of equiluminant red and green square textels $8.6^{\prime}$ each side, regularly arranged along opposite $45^{\circ}$ diagonals on an equiluminant yellow background, as shown in Figure 3.

\section{Observers}

Twenty-eight students (from high school to university graduate) with normal or corrected-to-normal vision participated in the experiments. They were dispatched in an experimental $(n=16 ; 5$ male; 
a

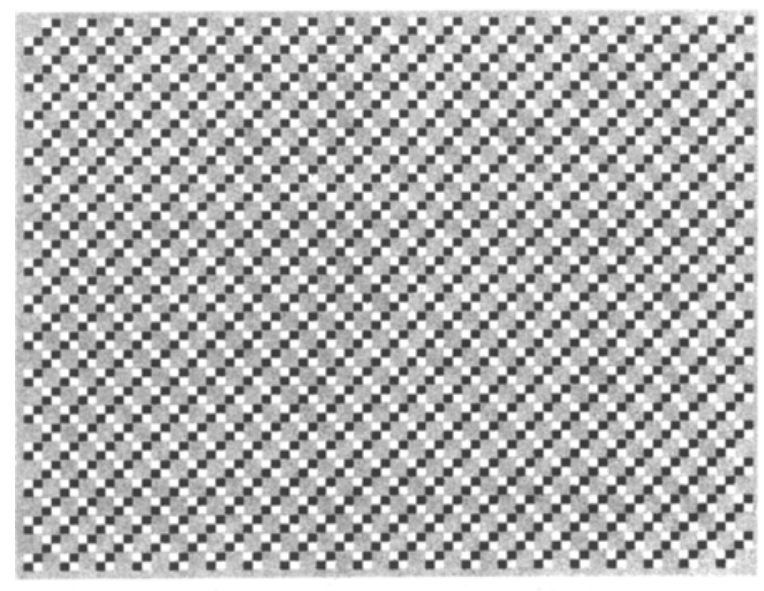

b

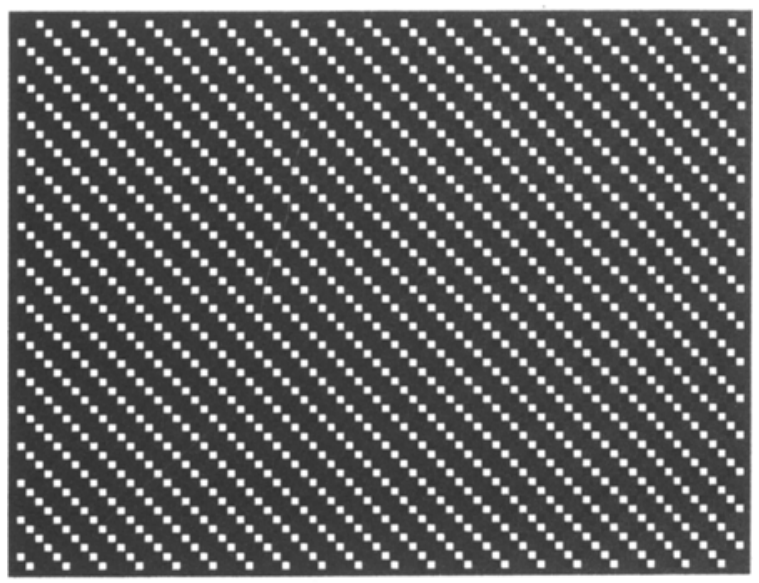

\section{Light}

\section{Dark}

Figure 3. Achromatic representations of the chromatic stimuli used to assess unique yellow. In the actual stimuli, dark and bright textels were equiluminant red and green, and the gray background was equiluminant yellow. (a) Brighter (more greenish) backgrounds yield a higher salience for the diagonal pattern of the dark (red) textels; (b) darker (more reddish) backgrounds reinforce the pattern of the bright (green) textels. The "unique gray" (unique yellow) is obtained when the patterns are equally visible.

age, 18-26) and a control ( $n=12 ; 8$ male; age, 19-25) group. They were all screened for normal chromatic vision with Ishihara plates. In addition, 3 of the 4 authors were run through the equivalentluminance-contrast procedure.

\section{Procedure}

Preliminary experiments. If performances obtained with edges defined by the double conjunction of color and orientation were to be compared to those defined by the double conjunction of lum!nance polarity and orientation, we needed to make sure that (1) the hues defining the texture elements (red and green) were equiluminant; (2) the red and green elements would yield equal chromatic contrasts relative to the background so that they would be equally salient (i.e., the hue of the equiluminant background had to be unique yellow); (3) the three types of contrast (red/green, dark/ bright, and $+45^{\circ}-45^{\circ}$ ) were equivalent to each other. As the results will show, a $\pm 45^{\circ}$ orientation contrast is less salıent than the maxImum obtainable red/green contrast. Given that a $\pm 45^{\circ}$ contrast is the largest achievable in the orientation domain, the only way of boosting its efficiency relative to the chromatic and/or luminance contrast would be to decrease the latter. However, lowering luminance or chromatic contrast might also reduce the perceived orientation contrast, so that the latter could not be equated to the former. As a consequence, the third requirement was satisfied for the chromatic and luminance contrasts only. The equivalent-contrast procedure was run with 3 of the 4 authors. Equlluminant and unique yellow points were assessed separately for each observer.

Equiluminance. The equiluminance assessment procedure involved a reverse-phi motion technique, originally described by Gorea et al. (1993). Equiluminance was achieved for each observer in two steps:

Step 1. The background was yellow and the bars were (1) yellow and red or (2) yellow and green. When the luminance of the red or green bars was equal to the luminance of the yellow background, the stımulus should yield $50 \%$ leftward and rightward directional reports (Gorea et al., 1993). Equiluminant settings were obtained by a staircase procedure that was designed to converge at $50 \%$ performance, and averaging the results of at least six such staircases.

Step $2 a, b$. The same procedure was repeated with the "unique yellow" background, after it was assessed separately for each observer (see below).

Unique yellow. This procedure was used to obtain a "unique yellow" background that was equidistant chromatically from the red and green elements; that is, it appeared neither reddish nor greenish (Raaijmakers \& de Weert, 1975). This was achieved by means of a staircase with the stimulus of Figure 3, where both textels and background were originally set at their equiluminant point, as assessed above. In each trial, observers were asked to report the most salıent perceived diagonal by pressing one of two response keys ("red" vs. "green" diagonal) after viewing the stimulus for $111 \mathrm{msec}$. The chromaticity of the background was varied along the red green ( $\mathrm{L}-\mathrm{M}$ ) chromatic axis so as to achieve $50 \%$ saliency for the redand green-defined diagonals. The staircase increased the relative contribution of the red phosphor to the background relative to that of the green phosphor subsequent to a "red" response, and decreased it after a "green" response, keeping the sum of the two contributions constant. The red/green values obtained for the last 10 reversals (out of 15 ) were averaged to obtain the unique yellow. This new yellow was used to reassess equiluminant red and green values, which were used in all subsequent experiments.

Equivalent-luminance contrast was measured in three steps for only 3 observers ( 3 authors). Single-feature texture edges were used in all experiments of this subsection.

Step 1. Percent signal threshold (see next section) was first assessed for the equiluminant color-defined texture edge stımulus.

Step 2. The obtained percent signal threshold for a given observer (hereafter referred to as $\theta$ ) was kept constant for the polaritydefined edge stimulus of Figures $2 \mathrm{a}$ and $2 \mathrm{~b}$, and discrimination performance for the latter was measured in terms of the luminance contrast required to achieve the same percent correct criterion as 
for the color stimulus. This luminance-contrast threshold was the luminance contrast equivalent to the red green chromatic contrast.

Step 3. $\theta$ was also measured for the chromatic orientationdefined texture edge stimulus. It was typically higher than the one obtained in Step 1. Because the orientation contrast was already maximal (i.e., $90^{\circ}$ ), it could not be increased further to achieve the same performance level, as for the color-defined edge.

Step 4. $\theta$ was finally measured with achromatic orientationdefined edge stimuli (Figures $2 \mathrm{c}$ and $2 \mathrm{~d}$ ), set at the equivalentluminance contrast obtained in Step 2. The obtained thresholds were practically identical to those obtained in Step 3, thus offering an additional validation of the equivalent-luminance-contrast procedure.

Percent signal thresholds $(\theta)$ were measured for both doubleconjunction and single-feature stimuli. The orientation of the texture parr edge (vertical or horizontal) was randomized across trials, and observers had to specify it by pressing one of two response keys. For any texture-pair type, the textels defining the texture edge were intermixed in variable proportions (noise [N]) so that the saliency of the edge was decreased. Take as an example the texture pairs of Figures $2 \mathrm{a}$ and $2 \mathrm{~b}$. A $0 \% \mathrm{~N}$ condition is the one in which all the bright textels are on the left side and all the dark ones are on the right side (Figure 2a). A $25^{\circ} \% \mathrm{~N}$ configuration is the one where onefourth randomly selected left-hand textels are exchanged with onefourth random right-hand textels (Figure $2 \mathrm{~b}$ ). A $50 \% \mathrm{~N}$ stimulus is a purely random one; that is, it yields no edge whatsoever. All data will be presented in terms of signal $(S)$, rather than noise, on a $0 \%$ to $100 \%$ scale, where $S=1-2 \mathrm{~N}$. A staircase procedure decreased $S$ by 2 percentage points after three consecutive correct responses, and increased it by the same amount after every wrong response, converging at $79^{\circ} \%$ correct. One session was completed after 20 reversals, and $\theta$ was computed as the average of the last 12 ones. This value of $\theta$ is the amount of signal that is necessary for the observer to perform the task at $79 \%$ correct.

Experimental design. A: Following the preliminary experiments, percent signal thresholds were obtained from all 28 observers for the four single-feature texture-pair conditions, twice for each condition per observer. This first stage was meant to familiarlze the observers with the texture-segregation task.

B: Observers were subsequently run through the doubleconjunction conditions in two distinct groups, the test group (which ran in two stages, $\mathrm{B} 1_{\text {TEST }}$ and $\mathrm{B} 2_{\text {TEST }}$ ), and the control group, which $\operatorname{ran} \mathrm{B}_{\mathrm{CTR}}$. In $\mathrm{B}_{\text {TEST }}$, the test group was presented with two color/ orientation and two luminance/orientation double-conjunction pairs with no other instruction than to specify the orientation of the texture edge. This will be referred hereafter as the precue phase. The four sessions were run in an $a b b a$ sequence for half of the observers and in a $b a a b$ sequence for the other half. In $\mathrm{B} 2_{\mathrm{TFST}}$, the construction principle of the double-conjunction texture pairs was explained to the observers. Attention was drawn to the fact that segregation might be much easier if they could "isolate" one subset of textels by attending to one of the defining features (i.e., a given color, luminance, or orientation) and perform the edge-orientation task based on the contrast of the second attribute. This will be referred to hereafter as the postcue phase. There were six possible "cues" "pay attention" to red, green, bright, dark, $+45^{\circ}$, or $-45^{\circ}$ elements. Given that the latter two occurred for both the color- and luminancedefined double conjunctions, there were eight cuing conditions overall. The cuing sequence was randomized according to two $8 \times 8$ Latin squares, one for each subgroup of 8 observers. Including the precue phase, each observer ran 12 sessions.

In $\mathrm{B}_{\mathrm{CR}}$, a control group was also run through 12 sessions but without any cuing or instructions as to the construction principle of the double-conjunction texture pairs. The sequence of color- and luminance-defined stimulı was randomized in such a way that each of the two stimuli was presented an equal number of times for each observer (i.e., 6). This control group was necessary to check for po- tential learning effects that could be confounded with the attentional cuing effect. In addition, the control group was also used to test the attentional/cuing effect across groups.

\section{RESULTS}

\section{Equivalent Luminance Contrast}

The 3 observers who ran this preliminary experiment (with single-feature texture pairs) yielded equivalentluminance contrasts of $38.5 \%, 36.4 \%$, and $43.6 \%$ at percent signal levels of $18.1,20.3$, and 18.6 , for observers T.V.P., T.E.C., and A.F., respectively. Because their luminance contrasts that were equivalent to the red/green chromatic contrast were very similar, their average was used for all 28 observers.

\section{Single-Feature-Defined Texture Edges}

Figure 4 displays mean $\theta$ s for the test (gray bars) and control (white bars) groups and for each of the four singlefeature texture pairs with the luminance contrast set at $40 \%$. As expected, the data show very similar thresholds for color- and luminance-defined edges, on the one hand, and for chromatic-orientation- and luminanceorientation-defined edges, on the other hand. Thresholds for the latter two are about a factor of 2 larger than for the former two. This was also the case for the 3 trained observers. Overall, the data show that, for the present stimuli, chromatic and luminance contrasts were equally efficient, while the orientation contrast was substantially less efficient.

\section{Cue Effects}

Figure 5 displays mean segregation thresholds $(\theta)$ with double-conjunction texture pairs for the control (bars with thin outline) and test (bars with thick outlines) groups. Thresholds for the color- and luminance-defined stimuli are shown as white and gray bars, respectively. The first four bars show $\theta$ s obtained during the first four sessions for both groups (the precue phase for the test group). The next eight $\theta$ s are for the test group in the postcue phase under all eight cuing conditions (as indicated on the abscissa: "R," cue on Red: "G," green; "B," bright; "D," dark; " /," $+45^{\circ}$; and "!," $-45^{\circ}$ ). The last two bars show $\theta$ s obtained by the control group averaged across the last eight sessions. Vertical lines are $\pm 1 S E$. The horizontal gray area indicates expected thresholds if observers had responded at chance level.

During the precue phase (first four sessions), performances of the test group for the two types of stimuli were barely below the gray horizontal bar--that is, slightly above chance level ( 14 out of 16 observers showed $\theta$ s $>98 \%$ ). For the luminance-defined double conjunctions, the control group also showed performances close to chance during both the first four and the last eight sessions. However, for this same group, color-defined stimuli yield significantly lower thresholds than for the luminancedefined ones during the 12 sessions of the experiment. 


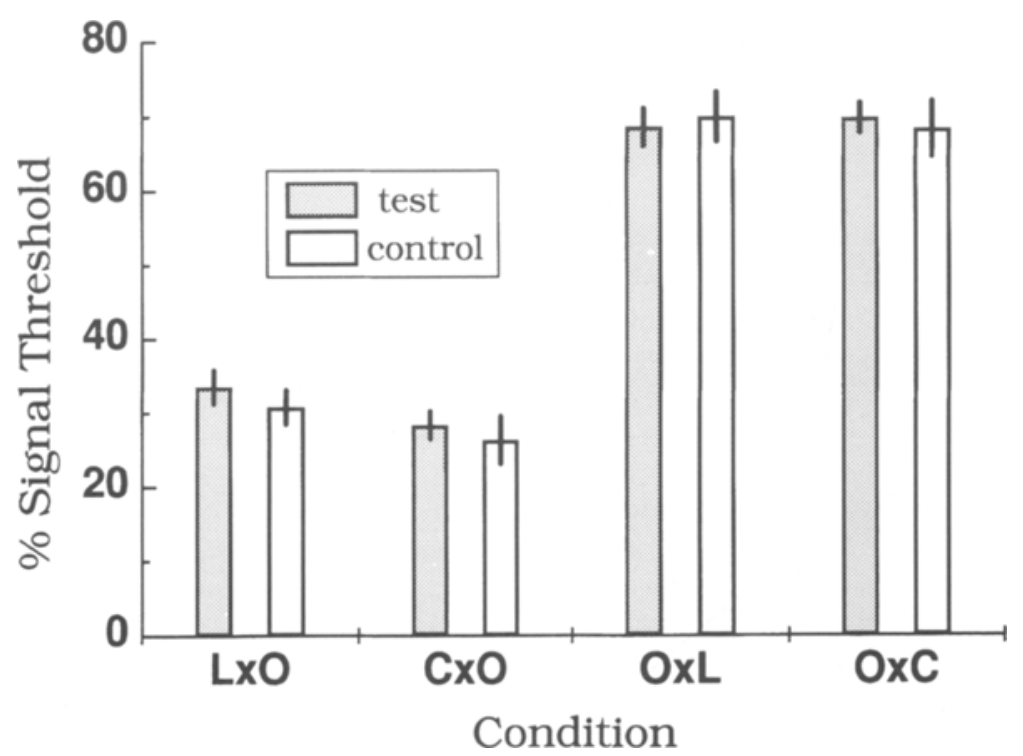

Figure 4. Percent signal thresholds for the test (gray columns) and control (white columns) groups with single-feature texture edges. Vertical bars show $\pm 1 S E$. Leftmost column pair: $L \times O$, luminance defined (Figures 2 a and $2 b$ ); second from left: $\mathrm{C} \times \mathrm{O}$, color defined; third from left: $\mathrm{O} \times \mathrm{L}$, achromatic orientation defined (Figures 2c and 2d); rightmost: $\mathrm{O} \times \mathbf{C}$, chromatic orientation defined.

At inspection, the data seem to display an overall cue effect both within and across groups. Cuing the colordefined stimuli appears to be substantially more efficient than cuing the luminance-defined stimuli. This is so despite the accurate match of their color and luminance contrasts. Within each group of stimuli, cuing either of its two defining attributes (orientation, color, or luminance) appears to have yielded similar effects despite the significantly lower efficiency of the orientation contrast. At first glance, cuing "red" seems more efficient than cuing "green," but this difference is not significant (see below).

Statistical analysis of the pre-/postcue effect. The design of the present experiments was by necessity unbalanced: The stimulus factor had two modalities (color

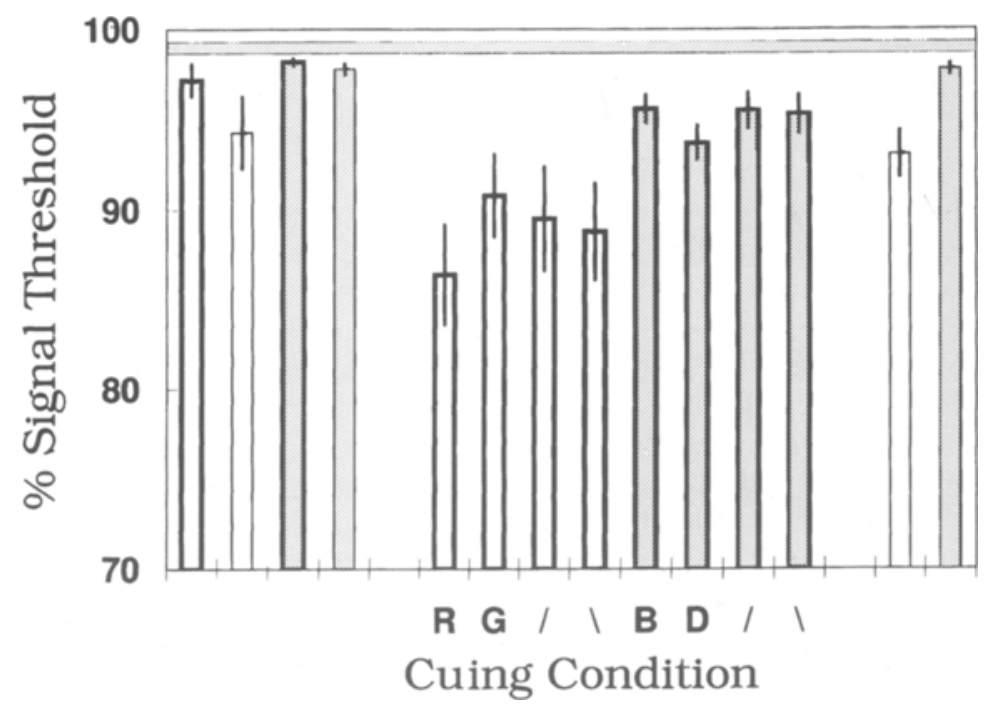

Figure 5. Mean percent signal thresholds with double-conjunction texture pairs for the test (columns with heavy outlines) and control (columns with thin outlines) groups, obtained during the first four (first four columns) and last eight (remaining columns) sessions. White and gray columns are for the chromatic and achromatic stimuli. Labels on the abscissa refer to the cued feature in the postcue phase of the test group: $R$, red; $G$, green; $B$, bright; $D$, dark; /, $+45^{\circ} ; 1,-45^{\circ}$. The last two columns represent the mean performance of the control group, averaged over the last eight sessions. Vertical bars show $\pm 1 S E$. 
and luminance) in the precue phase, and eight modalities (cue on red, green, dark, bright, and on $+45^{\circ}$ and $-45^{\circ}$ elements in both chromatic and achromatic stimuli) in the postcue phase. To circumvent this difficulty, two distinct analyses of variance (ANOVAs) were run independently. In the first ANOVA (Al), the eight cuing conditions were collapsed into two modalities - cuing the chromatic and cuing the achromatic stimulus-so that the analysis was of the 2 (stimuli) $\times 2$ (pre-/postcue) type. It was meant to assess the global cue and stimulus type effects. Al was performed both within the test group $\left(\mathrm{Al}_{\mathrm{W}}\right)$ and across the test and control groups $\left(A l_{A}\right) . A 1_{A}$ compared performances of the test group for the last eight sessions (i.e., postcue phase) with those of the control group, also during the last eight sessions. This design ensured that the two groups had the same exposure to the stimuli and was meant to test for significant differences in performance with and without cues. In order to avoid interference between the effects under test and a potential overall performance difference between the two groups, $A 1_{A}$ was run with normalized data, obtained for each observer by dividing all threshold scores by his/her mean threshold during the first four sessions. Normalization was performed independently for the color- and luminance-defined stimuli.

To analyze the specific cue effects, a second 2 (stimuli) $\times 2$ (cue type) ANOVA (A2) involved only performances of the test group in the postcue phase. Cue type referred to orientation $\left( \pm 45^{\circ}\right)$ and "polarity," each with two modalities. To achieve this format, color and luminance polarity cuing conditions were lumped together, "red" with "bright" and "green" with "dark" cuing, as well as "red" with "dark" and "green" with "bright" cuing. A2 was performed for each of these two grouping conditions. Because this grouping procedure precludes the comparison between the effects of cuing red versus green and of cuing dark versus bright elements, two independent one-way ANOVAs were run to this effect.

Global cue and stimulus effects. The within-group analysis $\left(\mathrm{A} 1_{\mathrm{W}}\right)$ confirmed all the observations made on mere inspection of Figure 5. It yielded significant effects of both the pre-/postcue manipulation $[F(1,15)=33.05$, $p<.00001]$ and stimulus type $[F(1,15)=6.47, p<.0225]$, as well as a strong interaction between the two factors $[F(1,15)=17.65, p<.0008]$. The significant interaction strengthens the conclusion that stimulus type modulates sensitivity only in the postcue phase. The across-groups analysis $\left(\mathrm{A}_{\mathrm{A}}\right)$ on the normalized data yielded the following effects: cue presence/absence $[F(1,26)=13.04$, $p<.001]$, stimulus type $[F(1,26)=11.88, p<.002]$, and an interaction $[F(1,26)=5.20, p<.031]$. The statistical significance of the performance differences between the groups is enhanced if we exclude 3 observers in the test group who could not achieve better-than-chance performance even after the stimulus construction was explained to them (see the Discussion section for more details on interobserver differences). Thus, both within- and across- groups analyses confirm the significance of the cue and stimulus effects as well as their interaction.

Specific cue effects (A2). As noted, this analysis was performed twice, once by pairing red-bright and greendark performances and a second time by using red-dark and green-bright pairing. The two variants yielded practically identical results (those of the second one are given in parentheses); the only significant effect was that of stimulus type $[F(1,15)=12.30(12.30), p<.0032]$. The two analyses showed no significant effect for either cue type (i.e., orientation vs. color or polarity), cue and stimulus type interaction, or the cued orientation $\left(+45^{\circ}\right.$ vs. $-45^{\circ}$ ). As noted, the format of the analysis did not allow assessing the effects of polarity (i.e., red vs. green and bright vs. dark cuing). The two one-way ANOVAs showed no such effect.

In conclusion, the statistical analysis shows (1) an overall pre-/postcue effect, (2) a larger effect for the color than for the luminance stimuli, (3) no effect of the cue type for a given stimulus type, (4) no interaction between stimulus and cue type factors, and (5) no effect between cuing bright versus dark or between cuing red versus green elements.

Practice effects. Figure 6 displays percent signal thresholds as a function of session order for both the test (a) and control (b) groups. Gray and cross-hatched columns show mean $\theta$ s for luminance- and color-defined stimuli, respectively. At inspection, mean $\theta$ s appear to have decreased slightly but systematically with session order for both groups. To assess the significance of practice, linear regressions were independently fit to performances obtained with luminance- and color-defined stimuli (solid and dashed lines in Figure 6) for each group. To avoid confounding the potential practice effect with the cue effect in the test group, only the last eight sessions (postcue phase) for this group were considered. For the control group, a session-order effect was obtained only for the color-defined stimuli (slope $=-0.63, p<$ .0004 ). The test group showed performance improvement over the last eight sessions for both color- (slope = -0.30 ) and luminance- (slope $=-0.18$ ) defined stimuli, but did not reach statistical significance. This observation suggests that once the observers have benefited from the cuing effect, they cannot improve significantly beyond that level.

\section{DISCUSSION}

The present experiments on double-conjunction texture segregation yielded two main effects, a feature-cuing effect and a practice effect, that were statistically significant, but weak. Feature cuing was effective for both colorand luminance-defined stimuli but less so for the latter (see analysis A2 in the Results section). This cuing efficiency difference was obtained despite the equivalence of the chromatic and luminance contrasts. Within each stimulus category (color- and luminance-defined), attending 

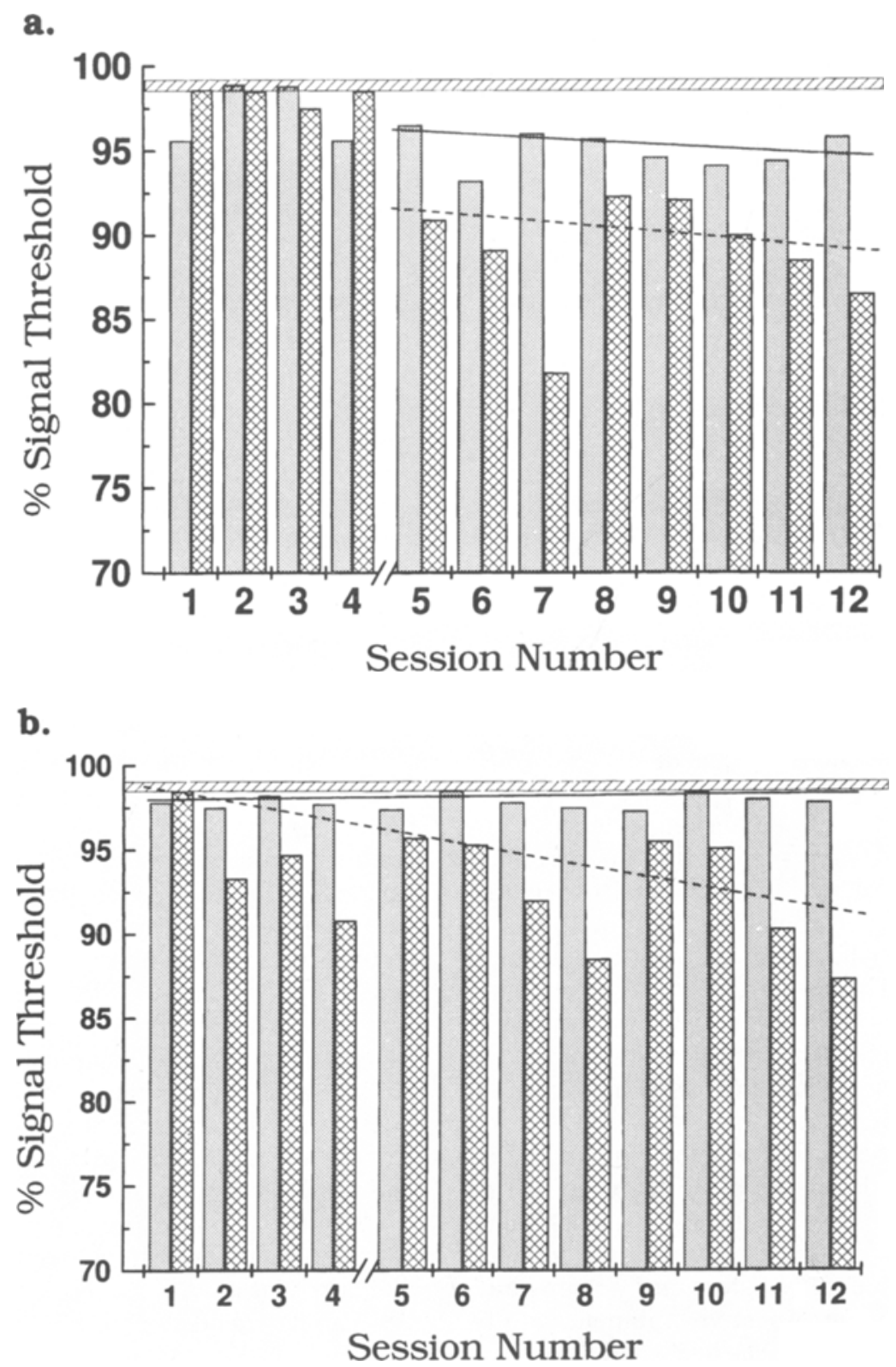

\begin{abstract}
Figure 6. Percent signal thresholds as a function of session order for the test (a) and control (b) groups. Gray and cross-hatched columns show $\theta$ s for luminance- and colordefined stimuli, respectively. Linear regression fits to the $\theta$ s obtained for the luminanceand color-defined stimuli are shown as solid and dashed lines, respectively. For the test group, linear regressions were fit only to the postcue phase $\theta$ s.
\end{abstract}

to either of its two defining dimensions (color/luminance or orientation) yielded equivalent performance enhancements despite a significant difference between the available contrasts along these dimensions (see Figure 4). Within each dimension, there was no significant difference between attending to one or the other of its two values ( red/green, dark/bright, $+45^{\circ} /-45^{\circ}$ ). Overall, the feature-cuing effects observed in this study with double- conjunction textures replicate those revealed by means of reaction time measures in visual search studies (Friedman-Hill \& Wolfe, 1995). It should be noted that this generalization is not necessarily self-evident, given both the differences in methodologies and the noted idiosyncrasies between visual search and texture-segregation performances (Wolfe, 1992). It must be noted that detecting the double-conjunction edge is a very difficult task. 
Even in the easiest case (color/orientation), the average signal threshold is $88.9 \%$ (Figure 5 , average of four postcue "color" columns), which is quite high when compared even with the most difficult single-feature taskorientation, with a signal threshold of $69.8 \%$ (Figure 4 , $\mathrm{O} \times \mathrm{C}$ column); this difference was obtained despite the shorter duration of the latter.

A global performance improvement occurred over time even without cuing (Figure 6b). Practice effects with double-conjunction stimuli have already been reported (Sireteanu \& Rettenbach, 1995; Von der Heydt \& Dürsteler, 1993), but their origin is not clearly understood. Interestingly, only the control group yielded a significant practice effect, and then only for the color/orientation double conjunction. This suggests that color features, as opposed to luminance ones, "draw attention" spontaneously so that naive observers may apply attentional strategies, consciously or not.

The overall cuing and practice effects observed in this study are not homogeneous across observers. In the test group some observers did not show any cue effect. In the control group, not all observers showed practice effects; some showed close to chance performance throughout the 12 experimental sessions. Friedman-Hill and Wolfe (1995) also reported interobserver differences in "guided" visual search experiments. The abrupt postcue performance improvement is very similar to the "Eureka effect" of Ahissar and Hochstein (1997), who reported significant improvement in perceptual learning for some observers after supplying them clear one-shot knowledge of the expected image. This improvement is also similar to the "abrupt learning" phenomenon of Rubin, Nakayama, and Shapley (1997). Interobserver differences suggest the involvement of inspection strategies and/or an uneven capacity of implementing them.

The fact of the matter is that double-conjunction texture pairs are practically impossible to segregate at first sight but, once the observer manages to group/isolate one of their defining features, their separating edge appears to be discernible, although it rarely "pops out" clearly. For some, this introspective argument is to be taken seriously: The clarity of the edge is, for them, convincing evidence that overt attention can selectively isolate sensory maps. ${ }^{4}$ However, upon closer examination, this line of argument leads to a debate. It has been argued that the difficulty of resolving some double-conjunction targets results from the absence of units simultaneously tuned to the two dimensions under focus (Nakayama \& Silverman, 1986). Another possibility suggested in the introduction was to pose their existence (see references in note 2 and Gorea \& Papathomas, 1993, for a review) and to attribute the difficulty of segregating double-conjunction stimuli to the noise from nonselective units. Performance enhancement contingent on a two-stage attentional strategy is then to be accounted for in terms of the suppression of this noise. Thus, contrary to intuition, the fact that "attending to red" followed by "segmenting on the basis of orientation" happens to be a useful strategy does not necessarily support a two-stage processing. "Attending to red" will have the disadvantage of ignoring a reliable edge signal from the "green-oriented map," but this disadvantage appears to be overcompensated by the suppression of the noise generated by the "chromatic nonspecific" and by the "green, nonoriented" units. Along this view, double-conjunction segmentation is a one-stage process even in an "attentive mode," involving the parallel inspection of several maps. In any case, the successful implementation by our observers of the suggested twostage processing strategy required selective processing along the two dimensions. The debate, as we see it, is whether or not these dimensions are coded directly by a single conjunctive mechanism, and our methods cannot distinguish between the two possibilities.

Also, the present experiments cannot discriminate between two proposals in the literature that genuinely fit into a two-stage processing scheme: Treisman's feature integration theory (Treisman, 1985; Treisman \& Gelade, 1980; Treisman \& Sato, 1990) and Wolfe's guided search model (Wolfe, 1992, 1994; Wolfe et al., 1989). In Treisman's model, color (nonoriented) and orientation (nonchromatic) responses lacking explicit spatial labeling (first stage) are "glued" or coupled together through focal attention (second stage) at the level of some disembodied spatial map. In Wolfe's model, the same first-stage onedimensional units do carry spatial labels and the doubleconjunction target is pointed to by the highest responsive location (second stage)- - that is, the one where the activated feature maps overlap spatially.

The statistical analysis shows a lesser cuing effect for the luminance- than for the color-defined stimuli. This is so despite the equivalence of the chromatic and luminance contrasts and despite the use of the same orientation contrasts in the two types of stimuli. One possibility is that the "noise" maps of luminance nonoriented and luminancepolarity nonspecific units cannot be suppressed through voluntary control. Another possibility is that chromatic maps can be attended more easily than luminance ones (Cavanagh, 1992).

Preliminary experiments were successful in equating the chromatic and luminance contrasts but not the orientation contrast. The data displayed in Figure 4 (singlefeature texture pairs) show that orientation-based segregation in both chromatic and luminance stimuli is more than a factor of 2 worse than luminance- or color-based segregation. On the other hand, within each doubleconjunction texture type, orientation and color/polarity cuing yielded identical discrimination performances. This contradiction is partly illusive. From the standpoint of a one-stage two-dimensional filtering formulation, it is clear that whatever the relative tuning properties of the front-end filter (along the two dimensions it codes- e.g., color and orientation), its overall output will be the same 
whether the edge is perceived as an orientation or as a color/luminance contrast. Instead, the only perceptual difference between a color- and an orientation-defined edge in a double-conjunction texture pair should reside in the specific population of suppressed or ignored units under each "attentive mode" (color- and orientation-insensitive units, respectively). Two-stage, one-dimensional filtering models should not predict a difference between the two attentive modes either. Within this framework, the overall probability of a correct segregation is the product of the probabilities of correctly grouping Attribute A (first stage) and then segmenting within the grouped subset, based on Attribute B (second stage). Under the assumption that performances with grouping and segregation are quite interrelated (to group the red textels, one must segregate them from the green ones), the overall probability would not change significantly if grouping was instead done on Attribute B and subsequent segregation on A.

Another explanation of why orientation and color cuing produced identical performances with double-conjunction texture pairs is that some observers did not obey the instructions strictly. It is possible that, once they were presented with the cuing strategies, they always used the one that they found most efficient (Duncan, personal communication, September 21,1997$)$. A related issue is that perhaps cuing facilitates a strategy to merely scrutinize a small number of items in the texture, which we shall term the "scrutiny" strategy (Wolfe, personal communication, September 21,1997$)$. Indeed, an experimenter is never sure what strategy an observer really uses, no matter how clear the instructions are, and a few observers may have used the "scrutiny" strategy (see item 4 below). We list some indications that most observers looked for a global texture edge: (1) Before embarking on the doubleconjunction texture sessions, they all experimented with the single-feature texture stimuli (Figure 2); thus, they were used to the task of looking for a texture edge. (2) When we explained the stimulus structure to them by showing them a figure similar to Figure $1 \mathrm{a}$, we instructed them to look for a global texture edge. (3) The manipulations of jagging and displacing the global edge seem to affect the scrutiny strategy more adversely than the strategy of detecting the global edge. (4) Finally, we conducted interviews with 9 observers who participated in the study and in pilot experiments. Only 2 of them reported using the scrutiny strategy; the rest reported that they looked at the stimuli globally to find the texture edge.

\section{REFERENCES}

AGONiE. C . \& GoREA. A. (1993). Equivalent luminance contrast of red-green drifting stımuli: Dependency on lumınance-color interactions and on the psychophysical task. Journal of the Optical Society of America A, 10, 1341-1352.

Ahissar. M., \& Hochstein, S. (1997). Task difficulty and the specificity of perceptual learning. Nature, 387, 401-406.

BorinG, E. G. (1950). A history of experimental psychology (2nd ed.). New York: Appleton-Century-Crofts.

Bravo, M. J., \& Nakayama, K. (1992). The role of attention in different visual-search tasks. Perception \& Psychophysics, 51, 465-472.
Cavanagh, P. (1992). Attention based motion perception. Science, 257, 1563-1565.

Davis, E. T., Kramer, P., \& Graham, N. (1983). Uncertainty about spatial frequency, spatial position, or contrast of visual patterns. Perception \& Psychophysics, 33, 20-28.

De ValoIs, R. L., \& DE ValoIs, K. K. (1988). Spatial vision. New York: Oxford University Press.

DoshER, B. A., \& LU, Z. L. (1997). Attention to location mediated by internal noise reduction. Investigative Ophthalmology \& Visual Science, 38(Suppl.), S687.

Duncan, J., \& Humphreys, G. W. (1992). Beyond the search surface: Visual search and attentional engagement. Journal of Experimental Psychology: Human Perception \& Performance, 18, 578-588.

Edwards, D. P., Purpura, K., \& Kaplan, E. (1995). Contrast sensitivity and spatial frequency response of primate cortical neurons in and around the cytochrome oxidase blobs. Vision Research, 35, 15011523.

Egeth, H. E., Virzl, R. A., \& Garbart, H. (1984). Searching for conjunctively defined targets. Journal of Experimental Psychology Human Perception \& Performance, 10, 32-39.

Friedman-HILL, S., \& WOLFE. J. W. (1995). Second-order parallel processing: Visual search for the odd item in a subset. Journal of Experimental Psychology: Human Perception \& Performance, 21, 531551.

Gorea, A., \& PaPathomas, T. V. (1989). Motion processing by chromatic and achromatic visual pathways. Journal of the Optical Society of America $A, 6,590-602$.

Gorea, A., \& Papathomas, T. V. (1991). Texture segregation by chromatic and achromatic visual pathways: An analogy with motion processing. Journal of the Optical Society of America A, 8, 386-393.

Gorea, A., \& Papathomas. T. V. (1993). Double-opponency as a generalized concept in texture segregation illustrated with color, luminance and orientation defined stimuli. Journal of the Optical Society of America A, 10, 1451-1462.

Gorea, A., Papathomas, T. V., \& Kovács, I. (1993). Motion perception with spatiotemporally matched chromatic and achromatic information reveals a "slow" and "fast" motion system. Vision Research, 33, 2515-2534.

GreEN, B. F., \& ANDERSEN, L. K. (1956). Color codıng in a visual search task. Journal of Experimental Psychology, 51, 19-24.

HumphrEYs, G. W. (1981). Flexibility of attention between stimulus dimensions. Perception \& Psychophysics, 30, 291-302.

Kingdom, F., Moulden, B., \& Collyer, S. (1992), A comparison between colour and luminance contrast in a spatial linking task. Vision Research, 32, 709-717.

Kramer, P., Graham, N., \& Yager, D. (1985). Simultaneous measurement of spatial-frequency summation and uncertainty effects. Journal of the Optical Society of America A, 2, 1533-1542.

LANDY, M. S., Maloney, L. T., Johnston, E. B., \& Young, M. (1995). Measurement and modeling of depth cue combination: In defense of weak fusion. Vision Research, 35, 389-412.

Leventhal, A. G., Thompson, K. G., Liv, D., Zhou, Y., \& Ault, S. J. (1995). Concomitant sensitivity to orientation, direction and color of cells in layers 2, 3 and 4 of monkey striate cortex. Journal of Neuroscience, 15, 1808-1818.

Livingstone, M. S., \& Hubel, D. H. (1984). Anatomy and physiology of a color system in the primate visual cortex. Journal of Neuroscience, 4, 309-356.

Maljkovic, V., \& Nakayama, K. (1994). Priming of pop-out: I. Role of features. Memory \& Cognition, 22, 657-672.

MCLeod. P., Driver, J., \& CRISP, J. (1988). Visual search for a conjunction of movement and form is parallel Nature, 332, 154-155.

Nakayama, K., \& Silverman, G. H. (1986). Serial and parallel processing of visual search conjunctions. Nature, 320, 264-265.

Palmer, J. (1994). Set-size effects in visual search: The effect of attention is independent of the stimulus for simple tasks. Vision Research, 34, 1703-1721

Papathomas, T. V., Kovács, I., Gorea, A., \& Julesz, B. (1995). A unified approach to the perception of motion, stereo and static-flow patterns. Behavior Research Methods, Instruments, \& Computers, 27, 419-432. 
PELLI, D. (1985). Uncertainty explains many aspects of visual contrast detection and discrimination. Journal of the Optical Society of America $A, 2,1508-1532$.

Quinl.AN, P. T., \& HUMPhreys, G. W. (1987). Visual search for targets defined by combinations of color, shape and size: An examination of the task constraints on feature and conjunction searches. Perception \& Psychophysics, 41, 455-472.

RaAijmakers, J. G. W., \& DE Weert, C. M M. (1975). Linear and nonlinear opponent color coding. Perception \& Psychophysics, 18, 474480.

Raymond, J., O'DONnell, H. L., \& Tipper, S. (1997). Attention modulates global motion sensitivity. Investigative Ophthalmology \& Visual Science, 38(Suppl.), S369.

Rubin, N., Nakayama, K., \& Shapley, R. (1997). Abrupt learning and retınal size specificity in Illusory contour perception. Current Biology, 7, 461-467.

ShiU, L. P., \& Pashler, H. (1994a). Neglıgible effect of spatial precuing on identification of single dots. Journal of Experimental Psychology Human Perception \& Performance, 20, 1037-1054.

Shiu, L. P., \& Pashler, H. (1994b). Spatial attention and vernier acuity. Vision Research, 35, 337-343.

Sireteanu, R., \& Rettenbach, R. (1995). "Early vision" is not so early after all. Texture segmentation in children and adolescents. Investigative Ophthalmology \& Visual Science, 36(Suppl.), S443.

SмIтн, S. L. (1962). Color coding and visual search. Journal of Experimental Psychology, 64, 434-440.

Treisman, A. (1985). Preattentive processing in vision. Computer Vision, Graphics \& Image Processing, 31, 156-1.77.

Treisman, A., \& Gelade, G. (1980). A feature integration theory of attention. Cognitive Psychology, 12, 97-136.

Treisman, A., \& Sato, S. (1990). Conjunction search revisited. Journal of Experimental Psychology Human Perception \& Performance, 16, 459-478.

Tsal, Y., \& Lavie, N. (1988). Attending to color and shape: The special role of location in selective visual processing. Perception \& Psychophysics, 44, 15-21.

Von der Heydt, R., \& Dürsteler, M. R. (1993). Visual search: Monkeys detect conjunctions as fast as features. Investigative Ophthalmology \& Visual Science, 34(Suppl.), 1288.

WOLFE, J. M. (1992). "Effortless" texture segmentation and "parallel" visual search are not the same. Vision Research, 32, 757-763.

WolfE, J. M. (1994). Guided Search 2: A revised model of visual search. Psychonomic Bulletin \& Review, 1, 202-238.

Wolfe, J. M., Cave, K. R., \& Franzel, S. L. (1989). Guided search:
An alternative to the feature integration model for visual search. Journal of Experimental Psychology: Human Perception \& Performance, $15,419-433$

\section{NOTES}

1. One should bear in mind that a double-conjunction task is not difficult by necessity. The difficulty of segregatıng the texture pair shown in Figure la can be manipulated by modifying any of its two defining (luminance and orientation) contrasts: If the luminance contrast is set to zero, the task is impossible; increasing this contrast will improve discrimınation at least up to some point, beyond which performance may etther saturate or possibly decrease again if the two conjoined attributes interact. Thus, for one to speculate on the putative differences among the neural mechanisms subserving the discrimination of a variety of double-conjunction stımuli, he/she must make sure that contrasts involved in each of these tasks are equivalent. Previous work different1ated between easy and difficult double-conjunction stımulı in search tasks and arrived at conclusions about different underlying processing modes (e.g., McLeod, Driver, \& Crısp, 1988; Nakayama \& Silverman, 1986; Quinlan \& Humphreys, 1987; Treisman \& Gelade, 1980; Wolfe, 1992; Wolfe et al., 1989), but did not consider such controls.

2. Other mapping segmentations have also been proposed (see the Discussion section and Wolfe, 1994, for a review).

3. For the chromatic double conjunction, the relevant maps should be "red" and "green." Physiological evidence of significant proportions of cells in VI and beyond that are nonspecific to a certain stimulus attribute (such as orientation, color, and/or luminance polarity) has been repeatedly reported (e.g., De Valoss \& De Valois, 1988; Edwards, Purpura, \& Kaplan, 1995; Leventhal, Thompson, Liu, Zhou, \& Ault, 1995, Livingstone \& Hubel, 1984).

4. This view has been suggested to us by Patrick Cavanagh. It is reminiscent of Titchener's and Wundt's views. For Titchener, the difference between attended and nonattended objects resides in the higher "clearness," "vividness," or "attensity" of the former. Wundt has formulated this difference in terms of two "degrees of consciousness" whereby attended objects "are brought within the focus of consciousness (Blickpunkt)" from within the more general "field of consciousness (Blickfeld)" (Boring, 1950, pp. 338, 415).

(Manuscript received June 23, 1997; revision accepted for publication July 7,1998 .) 